http://dx.doi.org/10.35381/racji.v5i8.590

\title{
Maternidad subrogada desde la perspectiva constitucional en el Ecuador
}

\section{Surrogacy from the constitutional perspective in Ecuador}

\author{
Karina Alexandra Espinoza-Campoverde \\ karina.espinoza@ucacue.edu.ec \\ Universidad Católica de Cuenca, Cuenca \\ Ecuador \\ https://orcid.org/0000-0002-4578-2654 \\ Juan Carlos Erazo-Álvarez \\ jcerazo@ucacue.edu.ec \\ Universidad Católica de Cuenca, Cuenca \\ Ecuador \\ https://orcid.org/0000-0001-6480-2270 \\ Diego Adrián Ormaza-Ávila \\ daormazaa@ucacue.edu.ec \\ Universidad Católica de Cuenca, Cuenca \\ Ecuador \\ https://orcid.org/0000-0002-3492-0943 \\ Cecilia Ivonne Narváez-Zurita \\ inarvaez@ucacue.edu.ec \\ Universidad Católica de Cuenca, Cuenca \\ Ecuador \\ https://orcid.org/0000-0002-7437-9880
}

Recibido: 13 de noviembre de 2019

Aprobado: 15 de diciembre de 2019

\section{RESUMEN}

En este artículo de revisión se analiza los preceptos constitucionales respecto de la maternidad subrogada debido a que existe la problemática que hay varios centros de salud en el Ecuador que se dedican al uso de la maternidad subrogada y ha surgido la necesidad de su regulación a pesar que existe una orden judicial que dispone normativizar esta actividad. Por ello, el objetivo es proponer la creación de una ley orgánica que garantice el derecho a la libertad sexual y la familia. La metodología aplicada parte del paradigma cualitativo, sustentado en métodos tales como análisis y síntesis bibliográficos, así como también el inductivo y deductivo. Se concluye que 
subsisten vacíos en el ordenamiento jurídico ecuatoriano puesto que no existe ninguna ley aprobada sino únicamente un proyecto de ley.

Descriptores: Técnicas de reproducción humana asistida; Madre subrogada; Madre gestante; Derecho a la libertad sexual; Derecho de familia.

\begin{abstract}
In this review article the constitutional precepts regarding surrogacy is analyzed, because there is a problem that there are several health centers in Ecuador that are dedicated to the use of surrogacy and the need for its regulation has arisen. Although there is a court order that regulates this activity. Therefore, the objective to be achieved is to propose the creation of a law. The research methodology starts from the qualitative paradigm, based on methods such as bibliographic analysis and synthesis, as well as the inductive and deductive. It is concluded that there are gaps in the Ecuadorian legal system since there is no approved law but only a bill.
\end{abstract}

Descriptors: Assisted reproduction techniques; Surrogate mother; Surrogate mother; Right to sexual freedom; Family right.

\title{
INTRODUCCIÓN
}

En el Ecuador durante los años 2007 a 2008, se efectuó un cambio trascendental en la organización del Estado, puesto que, se adoptó la creación de una nueva Constitución redactada por la Asamblea Constituyente en la ciudad de Alfaro, cantón Montecristi, provincia de Manabí que fue sometida a referéndum obteniendo una aceptación mayoritaria de los votos, y posteriormente entró en vigencia mediante la publicación del Registro Oficial No. 449 en fecha 20 de octubre del 2008 (Asamblea Constituyente del Ecuador, 2008).

La Carta Magna está compuesta por dos partes: una dogmática que a su vez, contiene los elementos constitutivos del Estado, derechos, y garantías jurisdiccionales; y otra parte orgánica que conlleva la organización del poder, organización territorial del Estado, régimen de desarrollo, régimen del buen vivir, relaciones internacionales y supremacía constitucional (Asamblea Constituyente del Ecuador, 2008). 
La Constitución de la República del Ecuador (CRE) en su primer artículo señala. "El Ecuador es un Estado constitucional de derechos y justicia ..." (Asamblea Nacional del Ecuador, 2008, art. 1) de una lectura ligera y rápida sobre el Estado Constitucional de Derechos, se podría pensar que hubo un error de codificación en el texto constitucional, sin embargo, éste calificativo es sumamente relevante e implica que los derechos son creaciones y limitan a todos los poderes tanto públicos como privados, además se crearon los mecanismos idóneos para hacer efectivos éstos derechos mediante las garantías constitucionales (Avila, 2008).

La CRE instituye un derecho constitucional que es el derecho a la libertad sexual situado dentro de los derechos de libertad, que reconoce y garantiza a las personas: “... El derecho a tomar decisiones libres, responsables e informadas sobre su salud y vida reproductiva y a decidir cuándo y cuantas hijas e hijos tener ..." (Asamblea Constituyente del Ecuador, 2008, art. 66 num 10) al respecto se entienden por derechos reproductivos como aquellos que permiten a los seres humanos a determinar con madurez el número de hijos a tener, en que momento y con qué individuo, determinando de esta manera su vida reproductiva determinando la capacidad de su vida reproductiva (Serrano, 2019).

A cerca de la libertad de poder decidir sobre tener hijos/as, se relaciona íntimamente con el Derecho a la Familia, que se encuentra reconocido en la norma suprema al proclamar: "Se reconoce la familia en sus diversos tipos. El Estado la protegerá como núcleo fundamental de la sociedad y garantizará condiciones que favorezcan integralmente la consecución de sus fines ..." (Asamblea Constituyente del Ecuador, 2008, art. 67)

Al respecto del Derecho a la Libertad Sexual y el Derecho a la Familia, la Corte Constitucional en el caso Satya, se ha pronunciado sobre las técnicas de reproducción asistida que son procedimientos para procrear, en virtud al ejercicio de su derecho de tomar decisiones libres sobre su vida reproductiva y decidir tener hijos, empleado el progreso científico en materia de procreación y formar una familia (Corte Constitucional del Ecuador, 2018). Dentro de este orden de ideas el progreso de la tecnología y ciencia ha sido impresionante en éstas últimas décadas, uno de los avances más importantes ha sido el desarrollo de la gestación por sustitución, 
que reemplaza al método tradicional de reproducción y permiten solucionar problemas de infertilidad del sexo femenino y masculino, cumpliendo así con el propósito de procreación.

Existen varios centros de salud de TRHA que se encuentran en funcionamiento en el Ecuador, sin embargo, al no existir una regulación jurídica dichos centros de salud se apegan a las disposiciones legales generales de salud y ética médica, y en virtud que en sus labores diarias tratan con un bien sagrado de todo ser humano que es el derecho a la vida, causan varios inconvenientes en los pacientes y posibles vulneraciones de los derechos del nuevo ser que está por nacer, es por eso que el problema de la presente investigación es: ¿Cómo elaborar la normativa jurídica empleando la técnica de reproducción de reproducción asistida de la gestación subrogada? De manera que el objetivo consiste en proponer una nueva Ley del Uso de la gestación por sustitución que garantice el Derecho a la Libertad Sexual y el Derecho a la Familia.

\section{DESARROLLO}

\section{EI Derecho a la Libertad Sexual y el Derecho a la Familia}

El Derecho interviene en la vida de los seres humanos desde el momento mismo en que somos fecundados en el vientre y seguimos amparados hasta el final de nuestros días y aún después, a través de nuestra descendencia. La vida puede ser vista desde distintas corrientes de pensamiento: jurídicas, biológicas, físicas, religiosas, sin embargo, lo que me ha interesado e intrigado en este proyecto de investigación es la concepción de un ser humano a través de la técnica de reproducción asistida de la maternidad subrogada y sus implicaciones en el derecho. El uso de las TRHA es cada día más frecuente y han traído como consecuencia que surjan dudas referidas a la filiación respecto de los padres e hijos, y se ha de analizar las siguientes interrogantes: ¿Quiénes deben ser considerados como progenitores? ¿Qué alcance tiene la donación de los gametos? ¿Tienen los donantes derechos filiatorios sobre el nuevo ser?, estas interrogantes serán contestadas a lo largo del desarrollo del presente trabajo de investigación. 
Las TRHA son aquellos procedimientos que en mayor o menor medida reemplazan o colaboran a uno o más pasos naturales del proceso de reproducción humana. Por regla general, una persona concibe una nueva vida mediante el acto sexual y hace uso de su derecho de reproducción sin la asistencia de terceros, no ocurre lo mismo cuando se trata de personas infértiles que deben ayudarse de las TRHA (Luna, 2008). Con el avance de la ciencia y tecnología dio como resultado las TRHA que son: inseminación artificial (IA), fertilización in vitro (FIV), criopreservación y subrogación (Méndez, 2014).

La IV es aquella técnica que radica en introducir el esperma previamente obtenido de un varón y colocarlo en el interior del útero de una mujer con el objetivo que exista una fecundación, consecuentemente se forme una nueva vida (Sanz, 2002); el FIV es el procedimiento realizado en un laboratorio mediante una placa de cultivo que fusionan el espermatozoide y óvulo para posteriormente implarlo en el útero, cuando aquello no se ha logrado de forma natural (Gómez, 1993); la criopreservación reside en extraer los gametos femeninos $y / 0$ masculinos y mantenerlos almacenados a temperaturas bajo cero para su posterior uso (Méndez, 2014).

La subrogación es un proceso médico donde se implanta el óvulo fecundado en el útero de la madre gestante o subrogada o portadora, quien se comprometerá a llevar a cabo el embarazo y parto hasta su culminación, para luego entregar el nuevo bebe a la pareja, esta TRHA se garantiza mediante la celebración de un contrato que puede ser a título gratuito u oneroso (Rosales, 2018).

La historia de la maternidad subrogada empieza en el año 1976, cuando el abogado Noel Kaene en Estados Unidos crea el término surrogate motherhood, para aplicarlo en la primera agencia de alquiler de vientres en los que se gestaban niños previo contrato con parejas interesadas (en este procedimiento se utilizó los óvulos propios de la mujer gestante fecundados con el semen del padre intencional), aquella mujer gestante cedía los derechos de maternidad del nuevo ser, después del parto a la pareja de esposos. En 1986 una madre de alquiler (madre gestante) gestó por primera vez un bebé sin relación genética alguna con ella (Ministerio de Salud Pública, 2017). 
De acuerdo con la investigación efectuada por la Comisión Nacional de Bioética en Salud del Ecuador (2018), afirma que se efectúan procedimientos de maternidad subrogada desde el año 1993, sin que en este lapso de tiempo exista una ley que regule dichos procedimientos. Adicional a ello, la Red Latinoamericana de reproducción asistida (REDLARA), asevera que existen siete centros de salud dedicados a la actividad económica del uso de las TRHA, que son: Biogepa centro de reproducción humana, CERHVALENCIA centro ecuatoriano de reproducción humana, clínica INFES, Concebir unidad de fertilidad y esterilidad, INNAIFEST centro nacional de reproducción asistida, Unidad de Fertilidad del Hospital Alcívar, Unidad de fertilidad del doctor Valdivieso (REDLARA, 2019)

El Diario El Telégrafo, afirma que, mediante las Técnicas de Reproducción Asistida, quince mil niños nacen en el Ecuador al año concebidos, en razón que varias parejas heterosexuales afrontan problemas de infertilidad y para ello, han encontrado una solución acudiendo a los centros especializados ubicados en las ciudades de Quito, Guayaquil y Cuenca (2018).

El Plan Nacional de Salud Sexual aprobado para los años 2017 a 2021 del Ecuador, promovido por la institución de salud pública delegada para el fomento del respeto e inclusión de los derechos humanos de libertad sexual y reproductivos enlista muchos inconvenientes de salud sexual y reproductiva y formula un plan de acción para evitar éstas dificultades, sin embargo, durante la revisión del documento no hace ninguna mención sobre las TRHA (2017).

En la experiencia como abogada en el libre ejercicio de la profesión, llegaron a consulta una pareja de esposos a fin de obtener información sobre la figura jurídica de la maternidad subrogada, dicha pareja de esposos, quienes tenían sus gametos femeninos (óvulos) y masculinos (espermatozoides) en buenas condiciones, sin embargo, la esposa sufría de una enfermedad comúnmente conocida como útero hostil, ellos habían intentado varios procedimientos médicos en la búsqueda de un nuevo hijo, sin obtener ningún resultado favorable, y siendo que una allegada a la pareja, se ofreció a ayudarles para gestar al bebe por nueve meses.

Indudablemente la pareja con ansias de tener un nuevo bebé enseguida acudió a un centro de salud especializado en reproducción asistida y efectivamente se realizó el 
procedimiento que fue todo un éxito, con el paso del tiempo la madre gestante comenzó a tener dudas sobre la entrega del nuevo ser y un día después del nacimiento del bebé, la madre gestante despareció con el bebe, sin que hasta la presente fecha se volviera a tener información alguna sobre el bebe o la madre gestante.

La maternidad tradicional consiste en el hecho que una mujer se convierte en madre del nuevo ser procreado, gesta aproximadamente los nueve meses y posterior da a luz, trayendo al mundo una nueva vida. En el procedimiento de la maternidad existe dos momentos: uno la gestación y el otro el parto (Parra, 2017). En relación a ello, la jurisprudencia de Colombia (1994), conceptualizo a la maternidad como: “... se entiende el acto de ser madre, y dicho acto supone una volición, es decir, un querer ser y una manifestación externa de ese querer" (p. 1).

De la gestación subrogada concurren dos periodos: uno es la fecundación en sí, que es la unión del óvulo con el espermatozoide y su implantación, y el otro es el nacimiento del nuevo ser, cuyo vínculo jurídico se concreta al momento de la aceptación como su hijo (Hernández y Santiago, 2011). Ahora bien, la maternidad en el ámbito del derecho es la figura jurídica de una mujer reconoce a su hijo como suyo, anteriormente se podía decir que los momentos de la maternidad que son la gestación y parto recaía sobre la misma persona, sin embargo, actualmente con las TRHA de la maternidad subrogada, puede darse que una es la madre intencional y otra la madre gestante.

\section{La maternidad subrogada y los derechos constitucionales}

La maternidad subrogada gira en torno de la evolución científica y los retos que representa ésta forma reproductiva para el derecho, que cuestiona la regla: "Mater Semper certa est, pater numquam" que se traduce como "la madre siempre es conocida, el padre nunca", haciendo referencia a la incuestionable prueba de identidad biológica relacionada directamente con la gestación (Ordeñana y Barahona, 2016), actualmente ésta regla, con la aparición de la figura de la subrogación en la maternidad, no es absoluta y es discutible. 
A cerca del concepto de maternidad subrogada es el suceso que vincula a una mujer para quedar embarazada y llevar a cabo la gestación a término alumbrando a un recién nacido por medio de un contrato a título gratuito u oneroso con otra persona que se convertirá en padre o madre de este nuevo ser. De esta manera la persona que se obliga para este proceso médico tendrá que ser apta para procrear y cede los derechos de este nuevo ser humano (Villamarín, 2014).

En relación al concepto de la maternidad subrogada puede legalizarse mediante un acuerdo de voluntades con o sin compensaciones, y que la futura madre subrogada se obliga a gestar al nuevo ser durante los nueve meses y/o donar su óvulo para la fecundación, comprometiéndose en entregar al recién nacido a los padres intencionales (Perez, 2002).

La gestación por sustitución es la técnica que una mujer pacta embarazarse con el objetivo de traer al mundo un niño que será cuidado por otras personas quienes legalmente serán identificados como sus padres, para el cumplimiento de esta obligación se puede efectivizar mediante un acuerdo de voluntades o contrato. Complementariamente el nuevo bebe será hijo biológico de la pareja intencional o subrogante y para ello, uno o ambos proporcionarán su material genético (Gimeno, 2011).

La pareja también llamada padres subrogantes o intencionales se utiliza para describir a los seres humanos que intentan ser los progenitores legales del nuevo ser producto de un contrato de maternidad subrogada, y de quienes se puede o no aportar su material genético y ser vinculados biológicamente con el nuevo ser (Frankford, Bennigton, y Ryan, 2015).

De lo anteriormente mencionado, se puede concluir que existe tres mujeres en el nacimiento del nuevo ser: 1) Comitente o intencional: es quien tiene la voluntad de procreación; 2) Biológica: es quien dona la cédula reproductora (óvulo) puede ser la identidad de la madre comitente, gestante o una donante anónima; y, 3) Gestante: es aquella mujer que lleva a cabo la gestación, quien adquiere el compromiso de realizar todos los esfuerzos necesarios para que el embrión llegue a desarrollarse correctamente y quien da a luz al nuevo ser, en una misma mujer puede recaer el tema biológico y la gestación (Ordeñana y Barahona, 2016). 
La gestación se clasifica en tradicional y gestacional. Aquella denominada tradicional es cuando una mujer accede a quedar embarazada mediante inseminación artificial cuyo material genético es del padre intencional, la madre gestante lleva a término los nueve meses del embarazo, aquí el nuevo ser tiene el material genético de su madre gestante y además del donador del semen, y los padres intencionales del bebe son los padres legales. Y la segunda, la subrogación gestacional se presenta cuando el material genético tanto del hombre como la mujer es donado y posteriormente implando mediante la fecundación in vitro en un laboratorio en la mujer gestante, aquí el nuevo ser tiene el ácido desoxirribonucleico de ambos padres intencionales (Rodríguez y Martínez, 2012).

El contrato de la subrogación se clasifica en altraista y comercial, en el primer caso al ser altruista no existe contraprestación o pago de dinero alguno con excepción de los gastos medicos y en el comercial, acuerdan las partes intervinientes en recibir una cantidad de dinero como pago para la realización de este contrato (Congreso de los Estados Unidos, 1989).

En esta investigación se referirá únicamente a la subrogación altruista que media un contrato o acuerdo de voluntades, pero no existen una compensación o pago económico y se la efectúa por diversos motivos como, por ejemplo: lazos de amor, relación de amistad o relaciones familiares, y aquí la pareja intencional aporta su propio material genético limitando a la madre subrogada a dos momentos que son el embarazo y parto (Hernández y Santiago, 2011).

La maternidad subrogada se relaciona con los derechos constitucionales como el derecho a la familia, derecho a la libertad sexual y derecho a la salud. En base a éste útlimo derecho de la salud, la maternidad subrogada debe contar con una permanente asistencia médica en todas sus dimensiones físicas y mentales, a fin de evitar daños previsibles y llevar a cabo una existosa gestación por sustitución (Ordeñana y Barahona, 2016).

La concepción de la familia tradicional o nuclear (padre, madre e hijos) ha ido evolucionando con el pasar de los tiempos, la norma suprema reconoce a la familia en sus diversos tipos y en relación a ello, la Corte Constitucional del Ecuador en su jurisprudencia fundada mediante el caso Satya y el matrimonio igualitario ha ido 
desarrollando el nuevo concepto de familia (Masabanda Analuiza, Aman Llerena, Montero Solano \& Analuiza, 2019)

El derecho de familia tradicionalmente es reconocido por la doctrina como familia nuclear, que es el grupo unido por vínculos biológicos, conformada por padre, madre e hijos, unidos por el vínculo matrimonial o de hecho (Ordeñana y Barahona, 2016), actualmente la norma suprema ha ido evolucionando y reconociendo los diversos tipos de familia mediante la jurisprudencia de la Corte Constitucional del Ecuador.

El artículo 67 de la Constitución del Ecuador, reconoce a las familias en sus diferentes formas y dice: “... El Estado la protegerá como núcleo fundamental de la sociedad y garantizará condiciones que favorezcan integralmente la consecución de sus fines ..." (Asamblea Constituyente del Ecuador, 2008, art. 67)

La Corte Constitucional mediante la sentencia de fecha 12 de junio del 2019, en el caso del matrimonio igualitario, resolvio que la opinión consultiva OC 24/17 sobre la Identidad de género e igualdad y no discriminación a parejas del mismo sexo, es vinculante ya que Ecuador es estado miembro de la Convención Americana de Derechos Humanos, y a través del bloque de constitucionalidad (convenios o tratados internacionales) forma parte del ordenamiento jurídico y lo más relevante de ésta sentencia fue que reconoce y protege el matrimonio igualitario entre parejas del mismo sexo (Corte Constitucional del Ecuador, 2019).

De ésta forma la norma suprema reconoce y garantiza la familia en diversos tipos, es decir, además del núcleo familiar tradicional conformado por padre, madre e hijo, también coexisten otros tipos de familias como lo son las familias: monoparentales (formadas por un padre e hijo o madre e hijo), homoparentales (padres o madres del mismo sexo con sus respectivos hijos), desintegradas (por razones de migración los progenitores se encuentran lejos y la familia se conforma con abuelo, nietos, entre otros) (Ordeñana y Barahona, 2016).

La Corte Constitucional en su sentencia No. 012-17-SIN-CC, ha determinado en cuanto a la familia lo siguiente que es aquel núcleo fundamental de la sociedad que genera conexiones jurídicas o de hecho y se instituye en el principio de igualdad de derechos y oportunidades a sus miembros, conjuntamente reconoce una concepción tradicional de la familia tradicional y sus tipos, instaurando el principio de 
corresponsabilidad a toda la familia, entendida ésta en sus diversos tipos (Corte Constitucional del Ecuador, 2017).

La Corte Constitucional del Ecuador en el caso Satya, ordenó mediante la reparación integral la medida de garantía de no repetición que al órgano legislativo en un el periodo máximo de un año contado desde el 29 de mayo del 2018, se emita una ley respecto de las TRHA conforme a los preceptos constitucionales, sin embargo, hasta la presente fecha no existe ningun proyecto de ley (Corte Constitucional del Ecuador, 2018).

El desarrollo de las técnicas de reproducción asistida abrió la posibilidad de la procreación conformando así una nueva diversidad de familia, puesto que, las personas que no podían conseguirlo de forma natural lo realizan a través de la ciencia y tecnología para concebir un nuevo ser. De ésta manera se logró la transferencia de herencia genética frente a la imposibilidad biológica, gracias a la aportación de gametos (masculinos y/o femeninos) por parte de donantes anónimos y mediante proceso de fecundación (Ordeñana y Barahona, 2016).

La Corte Constitucional denotó la importancia de las técnicas o procedimientos de reproducción asistida, identificandolas como un grupo de tratamientos médicos que se emplean para ayudar a las personas y parejas a lograr un embarazo. Estos procedimientos constituyen un importante campo en función que la procreación no solo involucra derechos personales y familiares, sino además el uso de material genérito tanto de los propios miembros de la familia como de posibles donadores, siendo notable una imperiosa necesidad de regulación legal apegada los preceptos constitucionales (Corte Constitucional del Ecuador, 2018).

En el proyecto al Código de la Salud, redacta un artículo sobre las TRHA y señala que se podrán realizar cumpliendo los principos bioéticos universales, con las normas, requisitos y regulaciones de la Autoridad Sanitaria Nacional y prohibe la contraprestación económica o compensaciones de cualquier tipo a cambio de gametos o embriones o subrogación del vientre, excepto los gastos médicos erogados por el tratamiento de uso de dichas técnicas (Asamblea Nacional del Ecuador, 2020). 
Otro derecho intimamente relacionado con la maternidad subrogada es el derecho a la libertad sexual, que está normativizado en la Constitución: “... el derecho a tomar decisiones libres, resposables e informadas sobre su salud y vida reproductiva y a decidir cuándo y cuántas hijas e hijos tener" (Asamblea Constituyente del Ecuador, 2008, art. 66 num. 10).

En cuanto a la libertad sexual, es la decisión de un persona respecto de procrear o no hijos, el número a tener y la planificación familiar, sin embargo, éste derecho crea polémica porque por un lado se sostiene que la libertad sexual es un derecho privado y propio de la persona, y por otro lado, se sustenta que el Estado como ente regulador debe normativizar éste derecho ya que se considera un tema de salud pública (Hernández y Santiago, 2011).

De acuerdo con lo manifestado en el párrafo anterior, en materia de procreación existe libertad, sin embargo, el Estado es responsable de garantizar los derechos constitucionales, y en base a ello, la Asamblea Nacional es el órgano cualificado para la creación de leyes, y tiene la obligación de normativizar la maternidad subrogada respetando el derecho constitucional de tomar decisiones libres respeto a la salud y vida reproductiva, así como el gozar de los beneficios y aplaicones del progreso científico (Corte Constitucional del Ecuador, 2018).

La jurisprudencia de la $\mathrm{CIDH}$, se ha pronunciado sobre el derecho a la libertad sexual, señalando que incluye el derecho a la autonomía reproductiva, derechos de la vida privada y la integridad personal, y a su vez se encuentra intimamente relacionado con el derecho a la salud. $Y$ hace énfasis que la falta de normativa legal en la salud reproductiva puede resultar en menoscabo grave del derecho a la autonomía y la libertad reproductiva (Corte Interamericana de Derechos Humanos, 2012).

La infertilidad o esterilidad del hombre o la mujer limita al derecho a la libertad, en específico al derecho al libre desarrollo de la personalidad, así como la efectividad de los derechos sexuales y reproductivos, situaciones que denotan la intervención del Estado como un ente regulador para la protección efectiva de estos derechos, quienes no pueden disponer de los medios económicos para acudir a un centro de salud privado haciendo uso de la TRHA. Los derechos sexuales y reproductivos se 
fundamentan de la libertad sexual y se debe incluir enn base al principio de igualdad (igualdad de información y acceso a los servicios de salud), en consecuencia el Estado debe actuar para faciliar el acceso a éste servicio (Lamas, 2001). A continuación se presenta la figura 1 que refleja que los derechos tutelados en la maternidad subrogada.

\section{TECNICA DE REPRODUCCION HUMANA ASISTIDA}

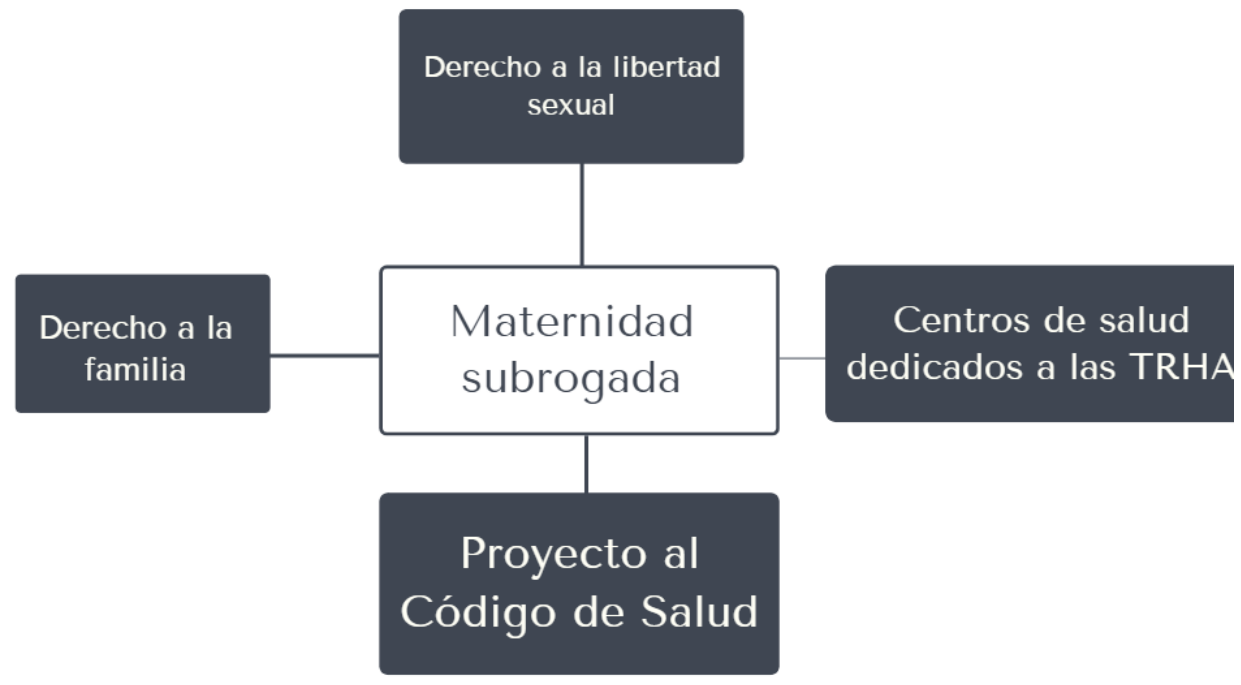

Figura 1 La técnica de reproducción humana asistida

\section{METODOLOGÍA}

Se utilizó el enfoque cualitativo mediante la recolección y análisis en una tipología documental - bibliográfica, desde la perspectiva descriptiva analizando aspectos sobre el problema. Los métodos aplicados fueron: Inductivo-deductivo, que ayudó para analizar la información teórica. Histórico - Lógico para levantar la información con el fin de conocer la situación de las variables y Analítico - sintético, que se pudo analizar algunas situaciones que afectan de manera directa a la problemática planteada.

La aplicación de todos estos métodos permitió analizar teorías, leyes, procedimientos judiciales, jurisprudencias, bibliografías, como parte de la población 
de estudio, generándose mediante el análisis documental jurídico, la construcción de aportes reflexivos teóricos en el campo del derecho constitucional (Espinoza, Narváez y Erazo, 2019).

\section{APORTES GENERADOS}

El avance de la tecnología y ciencia en el transcurso de los años vienen siendo acelerado y el derecho tiene la obligación de relacionar en forma armónica con estos sucesos, y uno de ellos es uso de las TRHA, que ha permitido a las parejas disfrutar plenamente de su derecho de libertad sexual y derecho a formar una familia. La clasificación de las TRHA es: inseminación artificial, fecundación in vitro, criopreservación y maternidad subrogada.

La TRHA que se analizó en este proyecto de investigación fue la gestación subrogada altruista, que gravita en un procedimiento de una mujer llamada gestante se obliga a cuidar del embarazo durante los nueve meses de un embrión que fue fecundado previamente con el material genético de una pareja más comúnmente llamada padres intencionales, y una vez que se da el alumbramiento, la madre gestante a través de un contrato de modalidad gratuito cede los derechos del nuevo ser a los padres intencionales.

La maternidad subrogada altruista debe contar con personal médico, psicológico y de un profesional en derecho. El personal médico del centro de salud serán los encargados de llevar a cabo el procedimiento de fecundación, control del embarazo y alumbramiento; por otro lado el personal en psicología brindará ayuda tanto a la madre gestante como a los padres intencionales para llevar con éxito el manejo de emociones del procedimiento de maternidad subrogada; y finalmente un profesional en el área de derecho, quien se ocupará del contrato a título gratuito para establecer las responsabilidades de cada parte interviniente, por ejemplo, los padres intencionales asumirán los costos generados en el procedimiento de fecundación y además los gastos durante el embarazo y alumbramiento, y la madre gestante se obligará a ceder los derechos constitucionales y legales del nuevo ser.

Los derechos constitucionales como son: el derecho a la libertad reproductiva y el derecho a la familia, a su vez originan derechos legales como son: la filiación, derecho de alimentos, patria potestad, tenencia, régimen de visitas, etc, éstos tienen 
que ser modificados en los respectivos cuerpos legales, puesto que, existe una nueva forma de procreación que es la voluntad procreacional de los padres intencionales.

En cuanto al derecho familiar, al tratarse de un grupo prioritario de atención como son los niños, niñas y adolescentes, es indispensable la intervención de un juez de la unidad judicial de familia, mujer, niñez y adolescencia para aprobar el contrato a título gratuito de la maternidad subrogada con la finalidad que proteja el interés superior del nuevo ser.

El marco jurídico de la utilización de la TRHA denominada gestación por sustitución deviene del derecho público y es de interés social, cuyo objetivo es el establecimiento y regulación de requisitos y formalidades para el procedimiento. En adición, en este tipo de acto no debe devengar ningún tipo de contraprestación o compensación económica entre las partes intervinientes salvo los erogados por los gastos médicos, con ello se evita el alquiler del útero con fines comerciales y que sea un acto desinteresado con la única finalidad que la pareja pueda tener descendencia.

\section{REFERENCIAS CONSULTADAS}

1. Asamblea Constituyente del Ecuador. (20 de octubre de 2008). Constitución de la República del Ecuador. Preámbulo. Alfaro, Montecristi, Ecuador: Registro Oficial No. 449.

2. Asamblea Nacional del Ecuador. (2020). Proyecto del Código a la Salud. Salud sexual y reproductiva. Quito, Pichincha, Ecuador: Asamblea Nacional del Ecuador.

3. Avila Santamaría, R. (2008). Ecuador Estador constitucional de derechos y justicia. En M. d. Humanos, La Constitución del 2008 en el contexto andino. Análisis desde la doctrina y el derecho comparado (págs. 19-38). Quito, Pichincha, Ecuador: V\&M Gráficas.

4. Congreso de los Estados Unidos. (1989). Código de Washington. Sección 26.16.210. Washington, Estados Unidos: Legislación del Estado de Washington. 
5. Corte Constitucional del Ecuador. (10 de mayo de 2017). Sentencia No. $012-$ 17-SIN-CC. Casos No. 0026-10-IN y 0052-16-IN Acumulados. Quito, Pichincha, Ecuador: Corte Constitucional del Ecuador.

6. Corte Constitucional del Ecuador. (29 de mayo de 2018). Sentencia No. 18418-SEP-CC. Caso No. 1692-12-EP. Quito, Pichincha, Ecuador: Corte Constitucional del Ecuador.

7. Corte Constitucional del Ecuador. (12 de junio de 2019). Caso No. 11-18-CN. Matrimonio igualitario. Quito, Pichincha, Ecuador: Corte Constitucional del Ecuador.

8. Corte Interamericana de Derechos Humanos. (28 de noviembre de 2012). Caso Artavia Murillo y otros, fecundación in vitro vs. Costa Rica. San José, Costa Rica: $\mathrm{CIDH}$.

9. Derecho a la maternidad, EXP. T-35245 (Dr. Vladimiro Naranjo Mesa 21 de julio de 1994).

10. El Telégrafo. (26 de mayo de 2018). 1500 niños nacen en Ecuador con técnicas de reproducción asistida. El Telégrafo.

11. Espinoza Ortega, A. A., Narváez Zurita, C. I., \& Erazo Álvarez, J. C. (2019). EI control gubernamental a través de la auditoría de gestión bajo los ejes de transparencia, rendición de cuentas y participación ciudadana. Cienciamatria, 545.

12. Frankford, D., Bennigton, L., \& Ryan, J. (2015). Womb Outsourcing: commercial surrogacy in India. American Journal of Maternal Child Nursing.

13. Gimeno Berbegal, A. (2011). El deseo de ser madre. Barcelona: Alba.

14. Hernández Ramirez, A., \& Santiago Figueroa, J. (septiembre de 2011). Ley de Maternidad Subrogada del Distrito Federal. Instituto de Investigaciones Jurídicas, 132, 1335-1348.

15. Lamas, M. (2001). Del cuerpo a la ciudadanía. El feminismo y la despenalización del aborto en México. Fondo de cultura económica, 221-239.

16. Luna, F. (2008). Reproducción asistida, género y derechos humanos en América Latina. San José, Costa Rica: Instituto Interamericano de Derechos Humanos.

17. Masabanda Analuiza, G., Aman Llerena, A., Montero Solano, J., \& Javier Analuiza, E. (2019). La evolución del derecho laboral de la mujer. Protección y fomento de la igualdad. IUSTITIA SOCIALIS, 4(7), 64-96. doi:http://dx.doi.org/10.35381/racji.v4i7.355 
18. Méndez Zambrano, M. (2014). La reproducción asistida. En V. Guerra, Y. Pérez, \& C. Lugo, Derecho Familiar Internacional, metodología para su estudio (Vol. I, pág. 1120). Medellín, Colombia: Biblioteca Jurídica Diké.

19. Ministerio de Salud Pública. (2017). Plan Nacional de Salud Sexual y Reproductiva 2017-2021. Ministerio de Salud Pública. Quito: Digital Center.

20. Ordeñana Sierra, T., \& Barahona Néjer, A. (2016). El Derecho de Familia en el nuevo paradigma constitucional. Quito, Pichincha, Ecuador: Cevallos Editora Jurídica.

21. Pacheco, V. (2018). Criterio y Recomendaciones Bioéticas sobre la maternidad subrogada en Ecuador. Quito, Pichincha, Ecuador: Ministerio de Salud Pública.

22. Parra Benitez, J. (2017). Derecho de Familia. Bogotá, Colombia: Editorial TEMIS Obras Jurídicas.

23. Perez Monge, M. (2002). La filiación de técnicas de reproducción asisitida. Madrid: Centro de estudios Registrales.

24. REDLARA. (2019). Red Latinoamericana de reproducción asistida. Obtenido de Centros de atención Ecuador: https://redlara.com/quem_somos.asp?MYPK3=Centros\&centro_pais=Ecuador

25. Rodríguez Yong, C. A., \& Martínez Muñoz, K. X. (2012). El contrato de maternidad subrogada: la experiencia estadounidense. Revista de Derecho, $X X V(2)$.

26. Rosales Andrade , V. E. (2018). La maternidad subrogada: propuesta para considerarla como una forma de trabajo en Ecuador. Law Review Universidad San Francisco de Quito, 140-156. Obtenido de file:///C:/Users/USUARIO/Downloads/1221-

Texto\%20del\%20art\%C3\%ADculo-3375-1-10-20180828.pdf

27. Sanz Alvarez, J. E. (2002). Fecundación Asistida. Ideas estructurales para la regulación de los métodos de procreación asistida. Medellín, Colombia: Grupo Editorial Ibañez.

28. Serrano Crespo, M. C. (2019). Apuntes sobre la reproducción asistida: una mirada desde la bioética a la situación normativa en Ecuador. Casa Editora, 90-124.

29. Villamarín Zuñiga, C. J. (2014). La maternidad subrogada en el Perú. Arequipa: Universidad Católica de Santa María. 
Año V. Vol. V. N ${ }^{\circ}$ 8. Enero - Junio 2020

Hecho el depósito de Ley: FA2016000064 ISSN: 2542-3371

FUNDACIÓN KOINONIA (F.K). Santa Ana de Coro, Venezuela;

Karina Alexandra Espinoza-Campoverde; Juan Carlos Erazo-Álvarez; Diego Adrián Ormaza-Ávila; Cecilia Ivonne Narváez-Zurita

(C2020 por los autores. Este artículo es de acceso abierto y distribuido según los términos y condiciones de la licencia Creative Commons Atribución-NoComercial-Compartirlgual 4.0 Internacional (CC BY-NC-SA 4.0) (https://creativecommons.org/licenses/by-nc-sa/4.0/). 